\title{
28 Resarch Soure \\ LncRNA CCAT1 Enhances Chemoresistance in Hepatocellular Carcinoma by Targeting QKI-5
}

\section{Jing Shi}

Jining Medical University

Cao Guo

Xiangya Hospital Central South University

Junli Ma ( $\nabla$ mjl7846@163.com )

Jining Medical University https://orcid.org/0000-0001-7233-5940

\section{Research}

Keywords: CCAT1, Hepatocellular carcinoma, Chemoresistance, QKI-5

Posted Date: August 20th, 2020

DOI: https://doi.org/10.21203/rs.3.rs-60542/v1

License: (1) This work is licensed under a Creative Commons Attribution 4.0 International License. Read Full License 


\section{Abstract}

Background: A major reason for treatment failure of cancer is acquisition of drug resistance. The specific mechanisms underlying hepatocellular carcinoma (HCC) chemoresistance need to be fully elucidated. IncRNAs involve in drug resistance in some cancers, however, the exact functions of IncRNA colon cancerassociated transcript 1 (CCAT1) in oxaliplatin resistance in HCC need to be elucidated.

Methods: Functional analysis of CCAT1 on oxaliplatin sensitivity was performed in HCC cell lines HCCLM3 and HepG2, and in a subcutaneous tumor model receiving OXA treatment. Furthermore, the downstream signaling targets of CCAT1 in HCC were explored.

Results: CCAT1 promoted HCC proliferation and reduced the apoptosis induced by oxaliplatin. Knockout of CCAT1 could increased chemosensitivity in vitro and in vivo. Further study found that QKI-5 was an important mediator and blocking of QKI-5/p38 MAPK signaling pathway enhanced oxaliplatin sensitivity.

Conclusions: CCAT1 promoted proliferation and oxaliplatin resistance by QKI-5/p38 MAPK signaling pathway in HCC. Targeting CCAT1 in combination with chemotherapeutics may be a promising alternative to reverse drug resistance in HCC treatment.

\section{Introduction}

As the fourth most fatal cancer worldwide, hepatocellular carcinoma (HCC) is still a major global health problem[1].The high mortality is due to late diagnosis with metastasis or poor hepatic reserve. The prognosis of HCC patients, especially those at advanced stages, remains poor. The treatment options are essentially palliative, including targeted therapy and systemic chemotherapy. Targeted drugs represented by sorafenib, which are clinically approved for the advanced or metastatic HCC, extend survival by very few months[2,3].The commonly used therapeutic regimens including adriamycin, 5-fluorouracil and platinum-containing drugs have been adjudged to be effective in a selected population of patients with advanced HCC[4]. However, a major reason for treatment failure is the acquisition of multi-drug resistance (MDR). The mechanisms involved in HCC intrinsic or acquired drug resistance are complex, such as drug efflux pumping, enhanced DNA damage repair capacity, inactivation of cell apoptosis and so on[5, 6]. However, the specific mechanisms underlying HCC drug resistance need to be fully elucidated.

Noncoding RNAs (ncRNAs), including long noncoding RNAs (IncRNAs), were found to be involved in biological processes, including proliferation, apoptosis, autophagy and metastasis by influencing gene expression at the transcriptional or post-transcriptional levels[7, 8]. Besides serving as biomarkers for diagnosis of many cancers, IncRNAs involve in drug resistance in multiple types of cancer including HCC[9]. Dysregulation of IncRNAs have been reported to be associated with drug resistance and radioresistance. LncRNA HULC was aberrantly expressed in HCC and significantly associated with oxaliplatin, 5-FU and pirarubicin sensitivity through USP22/Sirt1/autophagy pathway[10]. LncARSR was found to play a part in doxorubicin resistance of HCC. Overexpression of IncARSR promoted doxorubicin 
resistance via modulating PTEN-PI3K/Akt pathway[11]. IncRNA HANR contributed to the development of HCC and enhanced chemosensitivity to doxorubicin by binding to GSKIP[12].

Colon cancer-associated transcript 1 (CCAT1), located in 8q24.21, is a commonly amplified genomic area in colorectal cancer. Abnormally expressed CCAT1 in multiple types of cancer including colorectal cancer, breast cancer, squamous cell carcinomas and HCC was associated with aggressive malignancies[1316]. More importantly, CCAT1 was also regarded as a biomarker for drug resistance in some cancers. It was found that CCAT1 was involved in cisplatin, 5-fluorouracil and paclitaxel resistance[17-19]. However, little is known about the exact functions of CCAT1 in oxaliplatin resistance in HCC. In this study, we identified the possibility that CCAT1 enhanced chemoresistance of oxaliplatin and investigated the underlying mechanisms. We found that CCAT1 effectively reduced the chemosensitivity of oxaliplatin in HCC by quaking (QKI)-5/p38 MAPK signaling pathway, providing a new avenue for HCC therapy.

\section{Methods}

Cell lines and and transfection: HCC cell lines HCCLM3 and HepG2 were cultured in Dulbecco's modified Eagle's medium (DMEM, Gibco, Grand Island, NY) containing 10\% fetal bovine serum (FBS) in a cell incubator with $5 \% \mathrm{CO}_{2}$ at $37^{\circ} \mathrm{C}$. pcDNA-CCAT1 plasmid or empty vector was transfected into HCCLM3 and HepG2 cells by Lipofectamine ${ }^{T M} 3000$ (Invitrogen, Carlsbad, CA) reagent following the manufacturer's protocol. Knocked out CCAT1 in the above cells by CRISPR-CAS9. CRISPR guide RNA specifically targeting CCAT1 sequence (forward gRNA GCCCCTGGCCAACTATATCT; reverse gRNA ATTTGGTCATAATGCGGAAA) was constructed by Genechem (Shanghai, China) and transfected into the above cells.

qRT-PCR: Total RNA was extracted and reversely transcribed into CDNA using the Reverse Transcription System Kit (TaKaRa Bio Inc., Otsu, Japan). The levels of RNA expression were quantified by qRT-PCR assay (TaKaRa) and calculated by the $2^{-\triangle \Delta \mathrm{Ct}}$ method. The values were normalized to an endogenous control GAPDH.

Chemosensitivity detection: The HCC cells were plated in 96-well plates and exposed to the chemotherapeutic agent of oxaliplatin at the final concentrations of $0,0.1,1,10,100,1000 \mu \mathrm{M}$ the next day for 48 hours. Viable cells were determined by cell counting kit-8 (CCK-8, Dojindo, Kumamoto, Japan) according to the manufacturer's instructions. The cell survival rates and the dose-dependent curves of oxaliplatin were plotted, $50 \%$ growth inhibition $\left(\mathrm{IC}_{50}\right)$ values were calculated and analyzed by GraphPad Prism 5.0.

Apoptosis assay: The assay was conducted by using the Annexin V-FITC Apoptosis Detection Kit (BD Biosciences, San Jose, $C A$ ) according to the protocol. Briefly, the treated cells were prepared and incubated with $5 \mu \mathrm{L}$ Annexin V-FITC and $5 \mu \mathrm{L} \mathrm{PI}$ for $15 \mathrm{~min}$ at room temperature in the dark. The cell apoptosis was performed on a Canto II flow cytometry machine (BD Biosciences) within one hour. 
Colony formation assays: The treated HCC cells were seeded into $60 \mathrm{~mm}$ dishes with a density of 500 cells/dish and incubated for two weeks. Then the colonies were fixed with ice-cold methanol and stained with $0.1 \%$ crystal violet. The numbers of colonies containing more than 50 cells were calculated.

Immunofluorescence (IF) staining: The treated cells were fixed with $4 \%$ paraformaldehyde, permeabilized with $0.1 \%$ Triton $\mathrm{X}-100$, blocked with $2 \%$ bovine serum albumin and incubated with the primary antibodies against active Caspase 3 or QKI-5 at $4^{\circ} \mathrm{C}$ overnight. The corresponding Alexa Fluor-conjugated secondary antibodies (Life technology, Waltham, MA) were added, and the nuclei were counterstained by DAPI.

Detection of caspase-3 and caspase-7 activities: Oxaliplatin-treated cells were plated in 96-well opaque white plate. Caspase3/7 activities were determined by Caspase-Glo® 3/7 Assay kit (Promega, Madison, $\mathrm{WI}$ ) according to the manufacturer's instructions. The blank control, negative control and treated groups were added into $100 \mathrm{ul}$ reaction system respectively and cultured for $2 \mathrm{~h}$ to being detected the fluorescence value on Promega Glomax.

Western Blot: The protein were separated by sodium dodecyl sulfate polyacrylamide gel electrophoresis (SDS-PAGE) and transferred to PVDF membranes (Millipore, Bedford, MA). After being blocked with $5 \%$ skim milk in tris buffered saline (TBS)-Tween 20, the membrane were incubated with the primary antibodies, which are listed in Table S1, followed by HRP-conjugated secondary antibodies (Proteintech, Wuhan, China). The immunoblots were visualized by employing the enhanced chemiluminescence kit (Millipore) on a gel imaging analysis system (Tanon, Shanghai, China).

Cell fractionation assay: HCCLM3 and HepG2 cells were harvested and incubated with a lysis solution. After being centrifuged, the supernatant was used for assessing the cytoplasmic RNA, and the pellet was used for nuclear RNA extraction. GAPDH and U6 were used as cytosolic and nuclear markers respectively.

In vivo xenograft studies: Male BALB/c mice (4 weeks) were injected with HCCLM3 and HepG2 cells with CCAT1 knock out and received intraperitoneal injections of oxaliplatin $(0.8 \mathrm{mg} / \mathrm{kg} / \mathrm{w})$. The tumor growth curves were monitored and the final tumor sizes were calculated using the following formula: $\mathrm{V}\left(\mathrm{mm}^{3}\right)=$ $\left(L \times W^{2}\right) \times 0.5$ (L: Tumor length, W: Width). The animal experiments were approved by the Animal Use Committee of Jining Medical University and all mice were treated humanely.

Immunohistochemistry (IHC) and a TdT-mediated dUTP nick end-labeling (Tunel) assay: After deparaffinization, rehydration and antigen retrieval, the sections were incubated with the primary antibodies (Bcl-2, Active Caspase-3) at $4^{\circ} \mathrm{C}$ overnight, followed by the corresponding secondary antibodies. Tunel assay was applied according to the manufacturer's instructions to detect the apoptosis of HCC cells in subcutaneously xenograft tumors.

RNA pulldown assay: Briefly, cells were lysated and incubated with biotin (Bio)-labeled oligonucleotide probes. The beads were washed and boiled in SDS sample buffer, and the retrieved protein was was validated by standard Western blot analysis. 
Statistical analysis: All statistical analyses were performed with the GraphPad Prism Software (La Jolla, CA, USA). Student's $t$ test was used to analyze differences between two groups, and two-way ANOVA was applied when more than two groups were compared. $p$-values $<0.05$ was considered as statistically significant.

\section{Results}

CCAT1 modulated sensitivity of HCC cells to oxaliplatin. We observed that CCAT1 was significantly higher in oxaliplatin treated cells of HCCLM3 and HepG2 than their parental cells $(p<0.01$, Fig. 1A). To explore the association between CCAT 1 and the sensitivity of HCC cells to chemotherapy, the CCAT1 expressions were firstly knocked out in HCCLM3 and HepG2 by CRISPR-Cas9. Subsequently, these HCC cell lines with different CCAT1 expression levels were exposed to increased concentrations of oxaliplatin from $0.1 \mu \mathrm{M}$ to $1 \mathrm{mM}$ for $48 \mathrm{~h}$ to determine the cell viabilities and the IC50 values of oxaliplatin. The results indicated that, compared to the negative control group, knockout of CCAT1 in HCCLM3 showed greater oxaliplatin sensitivity, with lower IC50 values (IC50: 46.56 vs. $23.22 \mu \mathrm{M}, p<0.01$, Fig.1B). As shown in Fig.1C, HepG2 cells exhibited the seme trend (IC50: $27.98 v s .13 .28 \mu \mathrm{M}, p<0.01$ ). The results suggested that there might be a correlation between CCAT1 and oxaliplatin sensitivity in HCC. Then we explored the proliferation and apoptosis of HCCLM3 and HepG2 cells influenced by CCAT1. The colony formation assay demonstrated that CCAT1 knockout could attenuate the numbers of colonies in these two cells, compared to the control groups ( $p<0.01$, respectively. Fig.1D). The apoptosis assay showed that knockout of CCAT1 significantly increased the apoptosis of HCC cells both in HCCLM3 and HepG2 cells treated by oxaliplatin $(p<0.01$, Fig.1E). Furtherly, HCCLM3 and HepG2 cells were tested caspase-3 activation in presence of oxaliplatin at their respective IC50 concentrations. As shown in Figure 1F, the cells with CCAT1 knockout exhibited higher caspase- 3 activation levels $(p<0.01)$. Caspase-Glo ${ }^{\circledR} 3 / 7$ assay indicated that caspase- 3 and caspase-7 activities were significantly increased when CCAT1 was knocked out, as compared with the control groups both in HCCLM3 and HepG2 cells ( $p<0.05$, Fig.1G).

CCAT1 involved in HCC resistance to oxaliplatin in vivo. To further explore the effects of CCAT1 on HCC chemotherapy in vivo, the stably knocked-out HCCLM3 and HepG2 cells were used to construct the implantation model. The results indicated that the tumors in CCAT1 knockout groups grew at a significantly slower rate than that in the control groups both in HCCLM3 and HepG2. Five weeks after subcutaneous implantation, the average tumor volume in the KO-CCAT1 groups were significantly smaller than the control groups $\left(0.96 \pm 0.17 \mathrm{~cm}^{3} v s .0 .38 \pm 0.05 \mathrm{~cm}^{3} ; 0.40 \pm 0.06 \mathrm{~cm}^{3} v s .0 .20 \pm 0.05 \mathrm{~cm}^{3}, p<0.05\right.$, Fig.2A \& Fig.2B). Tumor cell apoptosis in the subcutaneous tumors tissue was detected by TUNEL staining. As shown in Figure 2C, apoptotic cells were significantly increased when CCAT1 was knocked out. The apoptotic rates of HCCLM3 cells in the groups of control and KO-CCAT1 were $4.53 \pm 0.67 \%$ and $12.30 \pm 0.59 \%(p<0.001)$ and in the HepG2 cells were $5.73 \pm 0.58 \%$ and $9.00 \pm 0.79 \%$ respectively $(p<0.05)$. With regard to the expression of apoptosis-related proteins, IHC of tumors tissue was applied. Higher active caspase- 3 and lower Bcl-2 expression levels were observed when CCAT1 was knocked out both in HCCLM3 and HepG2 cells. 
CCAT1 interacted with QKI-5. The subcellular localization of IncRNAs holds valuable clues to their molecular function. CCAT1 is exclusively positioned in the nucleus[20]. Through bioinformatic database of IncATLAS (https://Incatlas.crg.eu/), CCAT1 was observed to be located in nucleus in HepG2 cells (Fig.3A). We then examined the subcellular localization of CCAT1 by qRT-PCR and the results verified that it is mainly localized in the nucleus and may exerted its functions through interacting with the nuclear proteins (Fig.3B). LncRNAs could exert their functions through RNA-interacting proteins. Through bioinformatic analysis (http://rbpdb.ccbr.utoronto.ca/), we dentified a set of RNA binding proteins (RBPs) targeted by CCAT1, including QKI (Fig.3C). QKI-5 is the major isoform of QKIs and was found to be enriched in the nuclei of HCC cells by immunostaining assay and this was consistent with the localization of CCAT1 (Fig.3D). Further studies were performed to investigate the relationship between CCAT1 and QKI-5, and the results from RIP assay showed the enrichment of CCAT1 in the QKI-5immunoprecipitation. RNA pull-down assay also confirmed the interaction of CCAT1 and QKI-5 (Fig.3E).

\section{Overexpression of QKI-5 reversed oxaliplatin resistance via inhibiting the p38 MAPK signaling pathway. It} is suggested that QKI regulates genes expression through interaction with the quaking response element (QRE). Among the target genes containing at least one QRE, Ras is cancer-related and MAPK signaling pathway might involve in the regulation[21]. To investigate the major pathway modulated by QKI-5, we up-regulate QKI-5 by in HCCLM3 and HepG2 cells and detected the expression of JNK, ERK1/2 and p38 by Western blotting. The results indicated that the phosphorylation of p38 MAPK was decreased following QKI-5 overexpressed (Fig.4A). We found that CCAT1 promoted the phosphorylation of p38, while up-regulated QKI-5 could attenuate p38 phosphorylation level induced by CCAT1 (Fig.4B). Further study on exploration of oxaliplatin sensitivity was conducted by CCK-8 assay. Compared with the control group, the cell viability of cells transfected with CCAT1 was increased, and the drug sensitivity was dramatically decreased, while up-regulated of QKI-5 could reincrease oxaliplatin sensitivity, with lower IC50 values (IC50 values of HCCLM3: NC: $46.04 \pm 1.10 \mu \mathrm{M}$, CCAT1: $62.08 \pm 2.36 \mu \mathrm{M}, \mathrm{CCAT} 1+$ Control: $63.31 \pm 1.39 \mu \mathrm{M}$, CCAT1+pcDNA3.1-QKI-5: $46.89 \pm 2.95 \mu \mathrm{M}$; IC50 values of HepG2: NC: $27.49 \pm 1.40 \mu \mathrm{M}$, CCAT1: $38.768 \pm 0.90 \mu \mathrm{M}$, CCAT1+Control: $36.29 \pm 1.13 \mu \mathrm{M}, \mathrm{CCAT1}+$ pcDNA3.1-QKI-5: $27.97 \pm 0.85 \mu \mathrm{M}, p<$ 0.01 , Fig.4C). The colony formation assay demonstrated that CCAT1 increased the numbers of colonies, which could be inhibited by up-regulated of QKI-5 (Fig.4D). Furtherly, cells transfected with pcDNA3.1-QKI5 exhibited higher caspase-3 activation levels ( $p<0.01$, Fig.4E\&F). These results demonstrate that CCAT1 promoted proliferation and oxaliplatin resistance by QKI-5/p38 MAPK signaling pathway.

\section{Discussion}

Drug resistance is often activated at the same time when oxaliplatin exerts anti-growth effects. A series of IncRNAs play important roles in chemoresistance and tumor progression. The mechanisms underlying the role of IncRNAs in HCC drug resistance are complicated. Enhanced drug efflux caused by ATP-binding cassette (ABC) transporters is an important contributor of drug resistance. IncRNA NR2F1-AS1 is upregulated in oxaliplatin-resistant HCC cells and confer $\mathrm{HCC}$ resistance to oxaliplatin by targeting $A B C C 1$ [22]. Upregulation of IncRNAs VLDLR and H19 regulated drug sensitivity by induced the expression of ABCG2 and MDR1 $[23,24]$. The increased ability of DNA damage repair helps cancer cells bypassing 
the cytotoxicity of chemotherapy drugs, therefore the IncRNAs involved in genomic stability may can modulate drug sensitivity[25]. Xin Yin et al. evaluated the different IncRNA expression profile between oxaliplatin-sensitive and oxaliplatin-resistant HCC cells and identified 120 differentially expressed IncRNAs[26]. In our study, oxaliplatin resistance induced by CCAT1 in HCC is clarified for the first time. There are very few research on the involvement of CCAT1 in platinum-based chemotherapy. Hu B et al. demonstrated that CCAT1 contributed to DDP resistance by targeting miR-130a-3p/SOX4 and regulating ABCG2 expression[27]. Wang DY et al. suggested that CCAT1 promoted cisplatin-induced apoptosis via targeting CCAT1/miR-454/survivin axis[28]. In this study, we found that CCAT1 was significantly upregulated in oxaliplatin-resistant HCC cells, indicating that CCAT1 is implicated in oxaliplatin resistance in HCC. Knockdown of CCAT1 significantly increased the apoptosis of HCC cells and enhanced sensitivity to oxaliplatin and further study demonstrated the role of QKI-5 played in this process.

Various factors, including ncRNAs, could regulate QKI. QKIs is a member of the RNA-binding proteins, including QKI5, QKI6, and QKI7. Through binding with QRE, QKI regulates mRNA stability, RNA transportation and translational of target mRNAs. As a major isoform of QKIs, QKI-5 has a nuclear localization signal and is reported to be a tumour suppressor for many cancer types[29, 30].

Researches indicated that depletion of QKI-5 increases cell proliferation and metastasis in oral squamous cell carcinoma, non-small cell lung cancer, renal cell carcinoma and HCC[29, 31-33]. What's more, QKI might involve in drug sensitivity. Some studies showed that overexpression of QKI-5 could attenuate the toxic effect of doxorubicin by regulating circular RNAs[34, 35]. Fang Yu et al. demonstrated that 5-FU increased FOXO1 expression via inhibition of QKI and QKI-mediated FOXO1 contributing to the oncogenesis of breast carcinoma[36]. The roles of QKI in HCC remain to be fully elucidated. In the present study, we found that QKI-5 played an important role in CCAT1-induced chemoresistance. When QKI-5 was overexpressed, HCC cells were more sensitive to oxaliplatin and the apoptosis rate was increased.

\section{Conclusions}

In our investigation, we have established a signaling cascade involving CCAT1 and QKI-5 in the regulation of cell proliferation and the chemoresistance of HCC cells. Targeting IncRNAs in combination with chemotherapeutics may be a promising alternative to reverse drug resistance in cancer treatment.

\section{List Of Abbreviations}

CCAT1: Colon cancer-associated transcript 1, HCC: hepatocellular carcinoma, IncRNAs: long non-coding RNAs, qRT-PCR: quantitative real-time PCR, RBPs: RNA-binding proteins, quaking: QKI-5

\section{Declarations}

Ethics approval and consent to participate: This study was prospectively performed and approved by the institutional Ethics Committees of Jining Medical University (ethical approval number: 2018-FY-027) 
Consent for publication: The details/images/videos will be freely available on the internet and may be seen by the general public.

Availability of data and materials: The datasets used and/or analysed during the current study are available from the corresponding author on reasonable request.

Competing interests: The authors declare that they have no competing interests.

Funding: This study was supported by the grants from the Nature Science Foundation of Shandong Province (No. ZR2018LH014) and the National Nature Science Foundation of China (No. 81802467).

Authors' contributions: JS contributed to analysis and interpretation of data and drafting of the manuscript. CG contributed to acquisition of data and technical support. JM contributed to study concept and design, analysis and interpretation of data and drafting of the manuscript. All authors read and approved the final manuscript.

Acknowledgments: Not applicable.

\section{References}

1. Bray F, Ferlay J, Soerjomataram I, Siegel RL, Torre LA, Jemal A. Global cancer statistics 2018 : GLOBOCAN estimates of incidence and mortality worldwide for 36 cancers in 185 countries. CA Cancer J Clin. 2018;68(6):394-424.

2. Shen YC, Lin ZZ, Hsu CH, Hsu C, Shao YY, Cheng AL. Clinical trials in hepatocellular carcinoma: an update. Liver Cancer. 2013;2(3-4):345-64.

3. Mazzanti R, Arena U, Tassi R. Hepatocellular carcinoma: Where are we? World J Exp Med. 2016;6(1):21-36.

4. Kalyan A, Nimeiri H, Kulik L. Systemic therapy of hepatocellular carcinoma: current and promising. CLIN LIVER DIS. 2015;19(2):421-32.

5. Salehan MR, Morse HR. DNA damage repair and tolerance: a role in chemotherapeutic drug resistance. Br J Biomed Sci. 2013;70(1):31-40.

6. Butler EB, Zhao Y, Munoz-Pinedo C, Lu J, Tan M. Stalling the engine of resistance: targeting cancer metabolism to overcome therapeutic resistance. CANCER RES. 2013;73(9):2709-17.

7. Frankel LB, Lubas M, Lund AH. Emerging connections between RNA and autophagy. AUTOPHAGY. 2017;13(1):3-23.

8. Chi Y, Wang D, Wang J, Yu W, Yang J. Long Non-Coding RNA in the Pathogenesis of Cancers. CELLSBASEL. 2019; 8(9).

9. Wei L, Wang X, Lv L, Liu J, Xing H, Song Y, Xie M, Lei T, Zhang N, Yang M. The emerging role of microRNAs and long noncoding RNAs in drug resistance of hepatocellular carcinoma. MOL CANCER. 2019;18(1):147. 
10. Xiong H, Ni Z, He J, Jiang S, Li X, He J, Gong W, Zheng L, Chen S, Li B, Zhang N, Lyu X, Huang G, Chen $B$, Zhang Y, He F. LncRNA HULC triggers autophagy via stabilizing Sirt1 and attenuates the chemosensitivity of HCC cells. ONCOGENE. 2017;36(25):3528-40.

11. Li Y, Ye Y, Feng B, Qi Y. Long Noncoding RNA IncARSR Promotes Doxorubicin Resistance in Hepatocellular Carcinoma via Modulating PTEN-PI3K/Akt Pathway. J CELL BIOCHEM. 2017;118(12):4498-507.

12. Xiao J, Lv Y, Jin F, Liu Y, Ma Y, Xiong Y, Liu L, Zhang S, Sun Y, Tipoe GL, Hong A, Xing F, Wang X. LncRNA HANR Promotes Tumorigenesis and Increase of Chemoresistance in Hepatocellular Carcinoma. CELL PHYSIOL BIOCHEM. 2017;43(5):1926-38.

13. Abedini P, Fattahi A, Agah S, Talebi A, Beygi AH, Amini SM, Mirzaei A, Akbari A. Expression analysis of circulating plasma long noncoding RNAs in colorectal cancer: The relevance of IncRNAs ATB and CCAT1 as potential clinical hallmarks. J CELL PHYSIOL. 2019;234(12):22028-33.

14. Zhang XF, Liu T, Li Y, Li S. Overexpression of long non-coding RNA CCAT1 is a novel biomarker of poor prognosis in patients with breast cancer. Int J Clin Exp Pathol. 2015;8(8):9440-5.

15. Jiang $Y$, Jiang $Y Y$, Xie JJ, Mayakonda A, Hazawa M, Chen L, Xiao JF, Li CQ, Huang ML, Ding LW, Sun QY, Xu L, Kanojia D, Jeitany M, Deng JW, Liao LD, et al. Co-activation of super-enhancer-driven CCAT1 by TP63 and SOX2 promotes squamous cancer progression. NAT COMMUN. 2018;9(1):3619.

16. Deng L, Yang SB, Xu FF, Zhang JH. Long noncoding RNA CCAT1 promotes hepatocellular carcinoma progression by functioning as let-7 sponge. J Exp Clin Cancer Res. 2015;34:18.

17. Hu M, Zhang Q, Tian XH, Wang JL, Niu YX, Li G. IncRNA CCAT1 is a biomarker for the proliferation and drug resistance of esophageal cancer via the miR-143/PLK1/BUBR1 axis. Mol Carcinog. 2019;58(12):2207-17.

18. Yang C, Pan Y, Deng SP. Downregulation of IncRNA CCAT1 enhances 5-fluorouracil sensitivity in human colon cancer cells. BMC Mol Cell Biol. 2019;20(1):9.

19. Wang Q, Zhang W, Hao S. LncRNA CCAT1 modulates the sensitivity of paclitaxel in nasopharynx cancers cells via miR-181a/CPEB2 axis. CELL CYCLE. 2017;16(8):795-801.

20. Wang N, Yu Y, Xu B, Zhang M, Li Q, Miao L. Pivotal prognostic and diagnostic role of the long noncoding RNA colon cancerassociated transcript 1 expression in human cancer (Review). MOL MED REP. 2019;19(2):771-82.

21. Li K, Zhong S, Luo Y, Zou D, Li M, Li Y, Lu Y, Miao S, Wang L, Song W. A long noncoding RNA binding to QKI-5 regulates germ cell apoptosis via p38 MAPK signaling pathway. CELL DEATH DIS. 2019;10(10):699.

22. Huang $H$, Chen J, Ding CM, Jin X, Jia ZM, Peng J. LncRNA NR2F1-AS1 regulates hepatocellular carcinoma oxaliplatin resistance by targeting ABCC1 via miR-363. J CELL MOL MED. 2018;22(6):3238-45.

23. Takahashi K, Yan IK, Wood J, Haga H, Patel T. Involvement of extracellular vesicle long noncoding RNA (linc-VLDLR) in tumor cell responses to chemotherapy. MOL CANCER RES. 2014;12(10):137787. 
24. Tsang WP, Kwok TT. Riboregulator H19 induction of MDR1-associated drug resistance in human hepatocellular carcinoma cells. ONCOGENE. 2007;26(33):4877-81.

25. Renganathan A, Felley-Bosco E. Long Noncoding RNAs in Cancer and Therapeutic Potential. ADV EXP MED BIOL. 2017;1008:199-222.

26. Yin X, Zheng SS, Zhang L, Xie XY, Wang Y, Zhang BH, Wu W, Qiu S, Ren ZG. Identification of long noncoding RNA expression profile in oxaliplatin-resistant hepatocellular carcinoma cells. GENE. 2017;596:53-88.

27. Hu B, Zhang H, Wang Z, Zhang F, Wei H, Li L. LncRNA CCAT1/miR-130a-3p axis increases cisplatin resistance in non-small-cell lung cancer cell line by targeting SOX4. CANCER BIOL THER. 2017;18(12):974-83.

28. Wang DY, Li N, Cui YL. Long Non-coding RNA CCAT1 Sponges miR-454 to Promote Chemoresistance of Ovarian Cancer Cells to Cisplatin by Regulation of Surviving. CANCER RES TREAT. 2020;52(3):798-814.

29. Fu X, Feng Y. QKI-5 suppresses cyclin D1 expression and proliferation of oral squamous cell carcinoma cells via MAPK signalling pathway. Int J Oral Maxillofac Surg. 2015;44(5):562-7.

30. Zhao Y, Zhang G, Wei M, Lu X, Fu H, Feng F, Wang S, Lu W, Wu N, Lu Z, Yuan J. The tumor suppressing effects of QKI-5 in prostate cancer: a novel diagnostic and prognostic protein. CANCER BIOL THER. 2014;15(1):108-18.

31. Liang G, Meng W, Huang X, Zhu W, Yin C, Wang C, Fassan M, Yu Y, Kudo M, Xiao S, Zhao C, Zou P, Wang Y, Li X, Croce CM, Cui R. miR-196b-5p-mediated downregulation of TSPAN12 and GATA6 promotes tumor progression in non-small cell lung cancer. Proc Natl Acad Sci U S A. 2020;117(8):4347-57.

32. Zhang RL, Yang JP, Peng LX, Zheng LS, Xie P, Wang MY, Cao Y, Zhang ZL, Zhou FJ, Qian CN, Bao YX. RNA-binding protein QKI-5 inhibits the proliferation of clear cell renal cell carcinoma via posttranscriptional stabilization of RASA1 mRNA. CELL CYCLE. 2016;15(22):3094-104.

33. Han J, Meng J, Chen S, Wang X, Yin S, Zhang Q, Liu H, Qin R, Li Z, Zhong W, Zhang C, Zhang H, Tang Y, Lin T, Gao W, Zhang X, et al. YY1 Complex Promotes Quaking Expression via Super-Enhancer Binding during EMT of Hepatocellular Carcinoma. CANCER RES. 2019;79(7):1451-64.

34. Gupta SK, Garg A, Bar C, Chatterjee S, Foinquinos A, Milting H, Streckfuss-Bomeke K, Fiedler J, Thum T. Quaking Inhibits Doxorubicin-Mediated Cardiotoxicity Through Regulation of Cardiac Circular RNA Expression. CIRC RES. 2018;122(2):246-54.

35. Yan F, Liu R, Zhuang X, Li R, Shi H, Gao X. Salidroside Attenuates Doxorubicin-Induced Cardiac Dysfunction Partially Through Activation of QKI/Fox01 Pathway. J Cardiovasc Transl Res. 2020.

36. Yu F, Jin L, Yang G, Ji L, Wang F, Lu Z. Post-transcriptional repression of FOXO1 by QKI results in low levels of FOXO1 expression in breast cancer cells. ONCOL REP. 2014;31(3):1459-65.

\section{Figures}


A
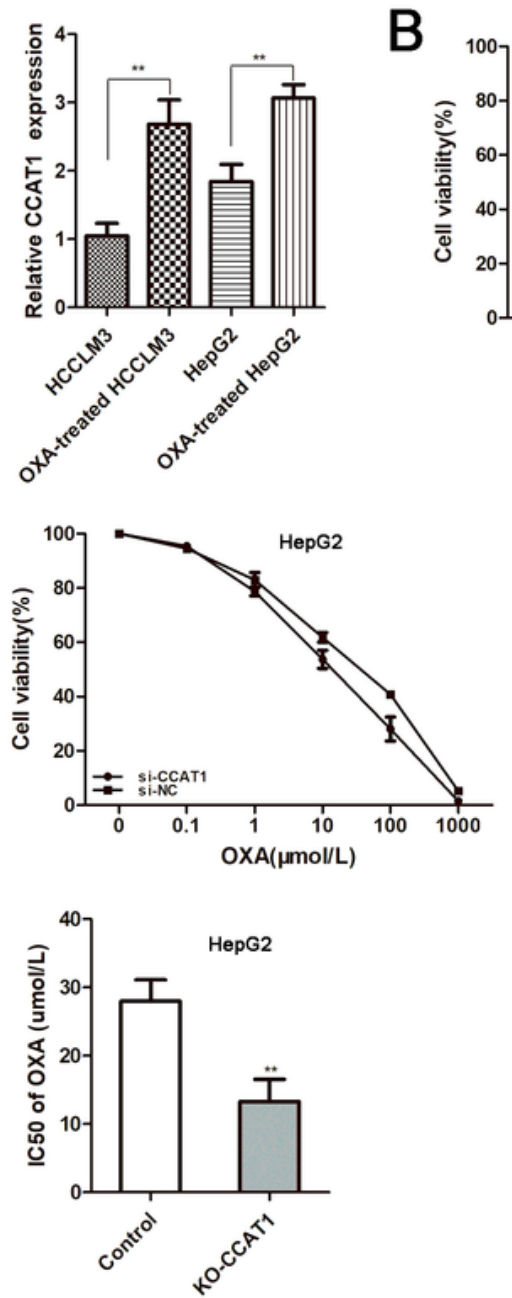

$\mathrm{F}$
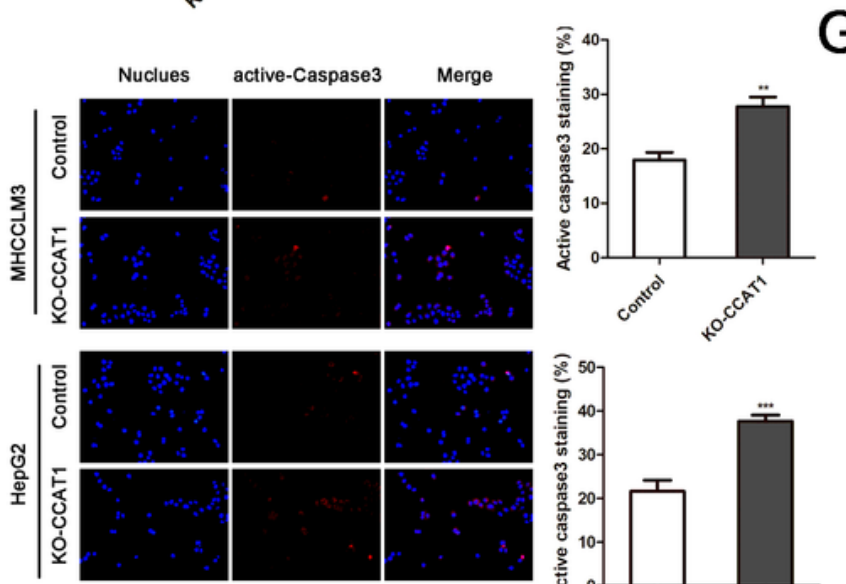

B

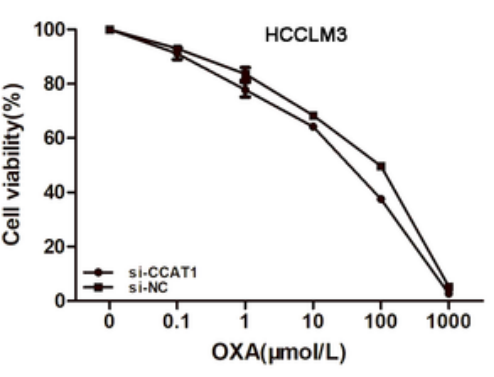

D

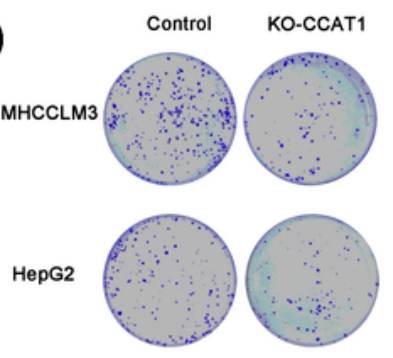

$\mathrm{E}$
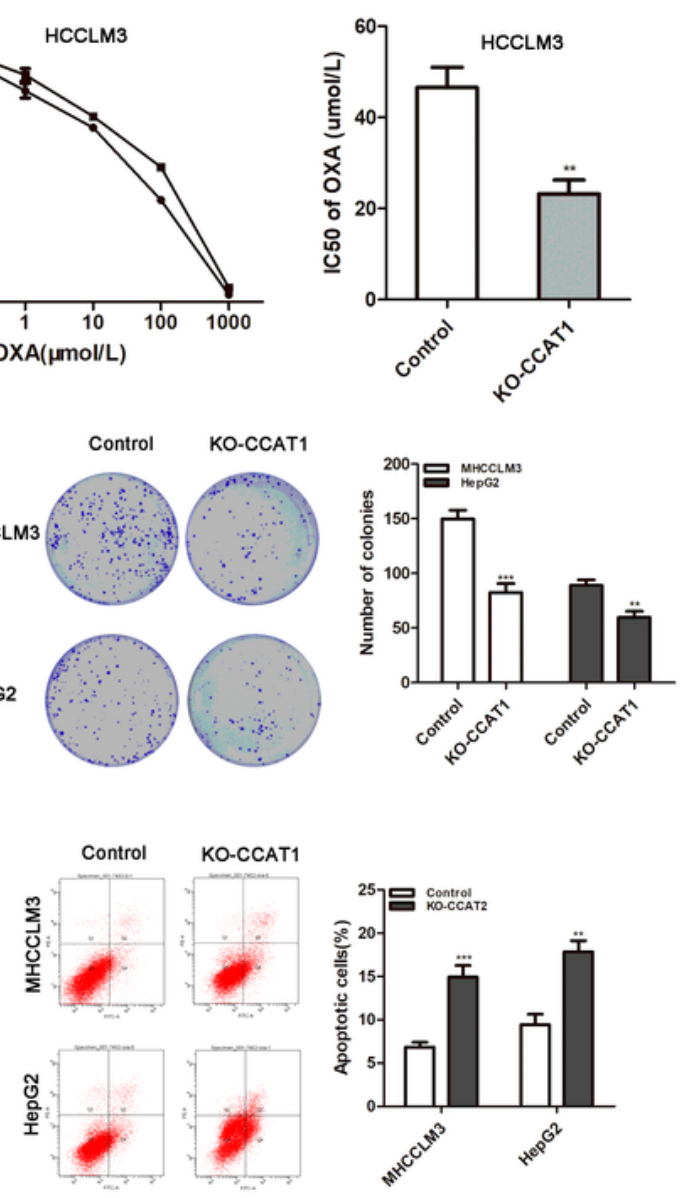
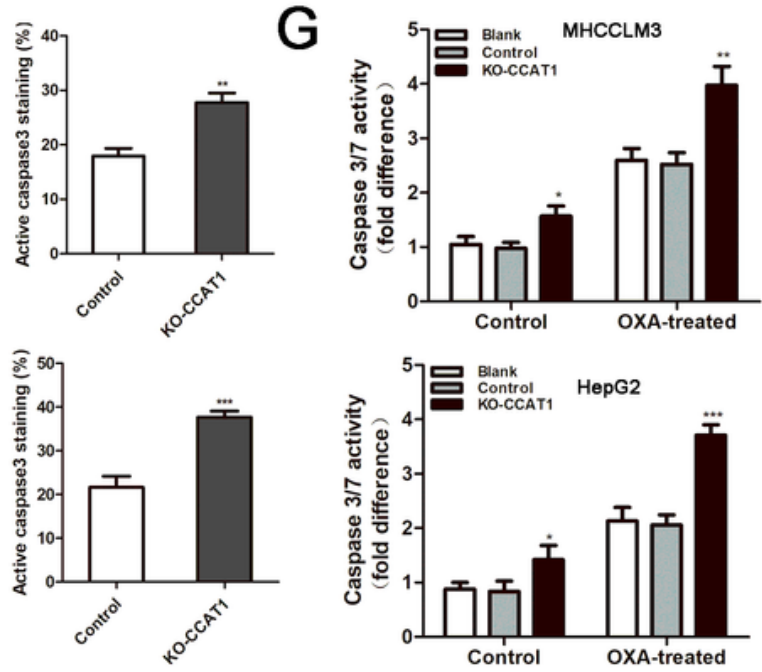

\section{Figure 1}

CCAT1 enhanced HCC resistance to oxaliplatin in vitro. (A) HCC cells received OXA treatment for $48 \mathrm{~h}$, the expression of CCAT1 was determined by qRT-PCR. (B\&C) HCCLM3 and HepG2 HCC cell lines were knocked out of CCAT1 and exposed to increasing concentrations of oxaliplatin from $0.1 \mu \mathrm{M}$ to $1 \mathrm{mM}$ for $48 \mathrm{~h}$ to determine the IC50 values by CCK-8 assay. (IC50 of HCCLM3 control and KO-CCAT1: 46.56 vs. 23.22 $\mu \mathrm{M}$; HepG2 control and KO-CCAT1: IC50: 27.98 vs. $13.28 \mu \mathrm{M}, \mathrm{p}<0.01$ respectively). (D) The colony 
formation assay showed that the numbers of colonies were reduced when CCAT1 was knocked out. (E) Significantly increased proportion of apoptotic cells by in the KO-CCAT1 groups. (F) Significantly increased caspase-3 activation following knock out of CCAT1, indicated by red fluorescence staining ( $p<$ 0.01). (D) Caspase-3 and caspase-7 activities were measured by Caspase-Glo® $3 / 7$ Assay $(p<0.05)$.
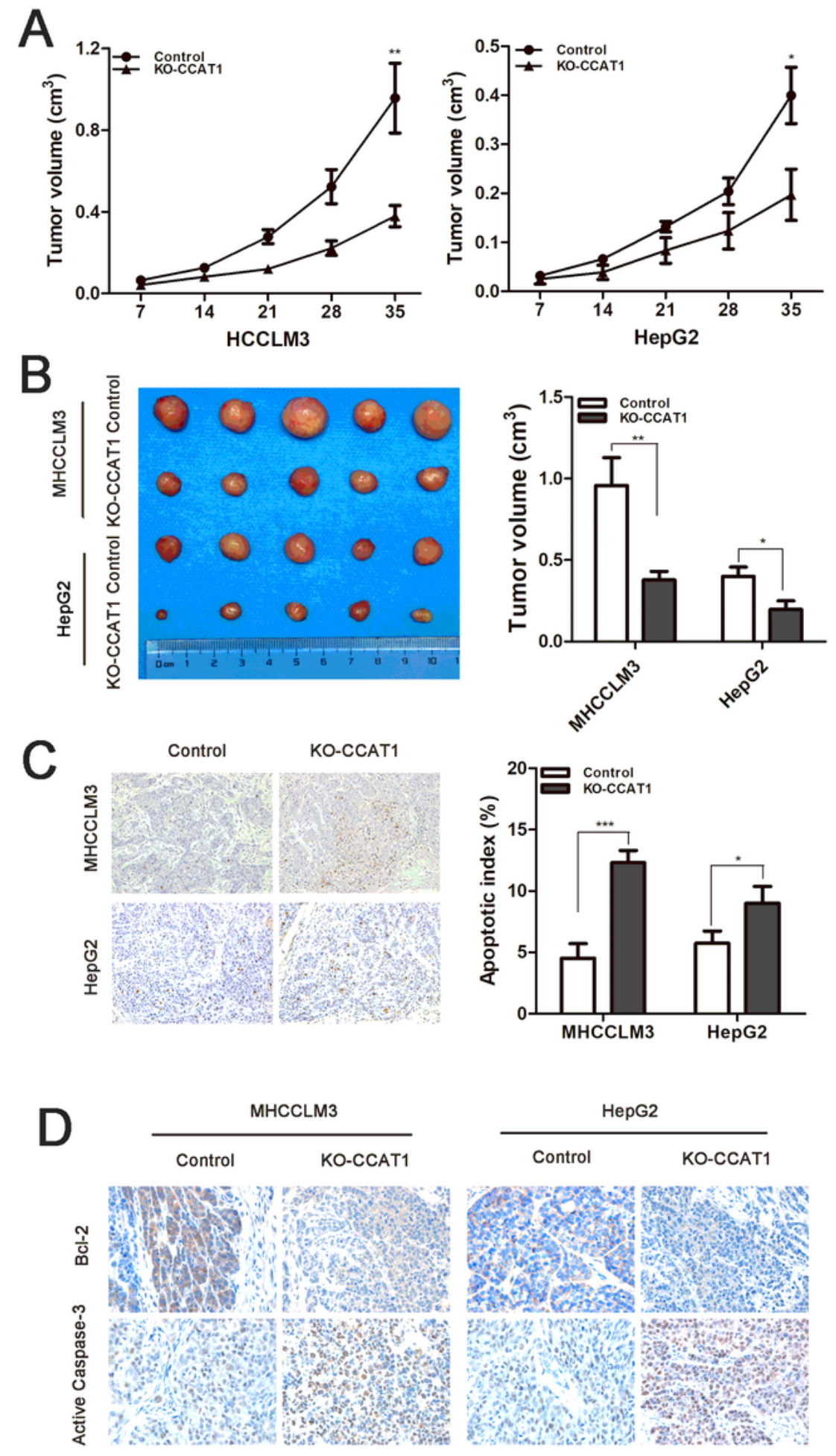

Figure 2 
CCAT1 involved in HCC resistance to oxaliplatin in vivo. (A) Tumor growth curves of subcutaneous implantation models of HCC. (B) The average tumor volumes were calculated using the following formula: $\mathrm{V}(\mathrm{mm} 3)=(\mathrm{L} \times \mathrm{W} 2) \times 0.5$ (L: tumor length, $\mathrm{W}$ : width). (C) TUNEL assay was applied to detect HCC apoptosis in subcutaneous implantation tumor (Original magnification: $\times 200)$. (D) Immunohistochemistry staining for Bel-2 and active caspase-3 expressions in subcutaneous tumor (Original magnification: $\times$ 400). *: $\mathrm{P}<0.05, * *: \mathrm{P}<0.01$, ***: $\mathrm{P}<0.001$.

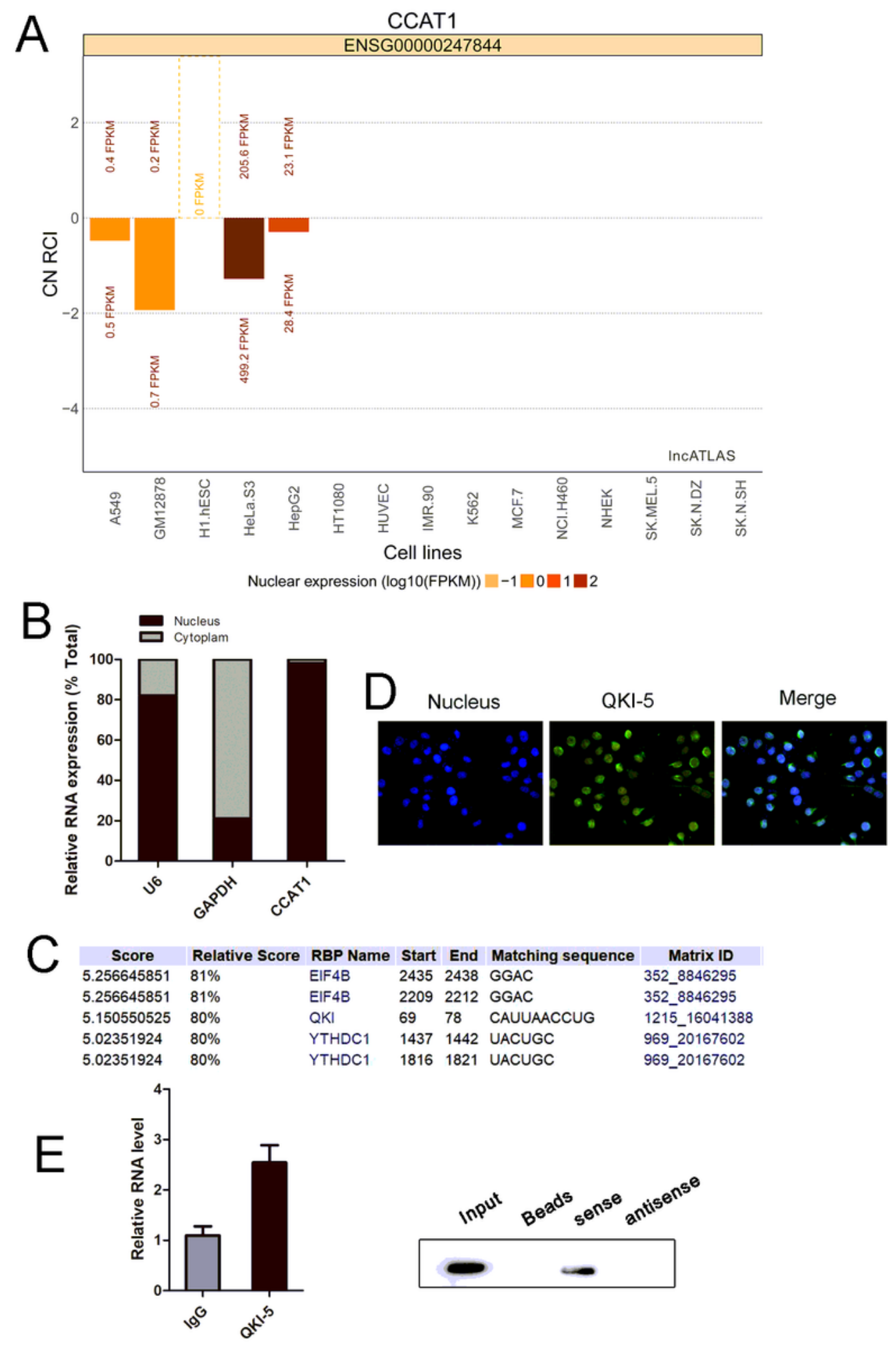

Figure 3 
CCAT1 interacted with QKI-5. (A) Subcellular localization plots displayed by IncATLAS. (B) This nuclear location was confirmed by the cytoplasmic and nuclear extracts measured from qRT-PCR. (C) RNAbinding proteins of CCAT1 was predicted by RBPDB (http://rbpdb.ccbr.utoronto.ca/) database. (D) Immunohistochemistry staining indicated that QKI-5 was located in the nucleus. (E) CCAT1 was enriched with QKI-5-immunoprecipitation. RNA pull-down assay was applied to detect the QKI-5 antibody.
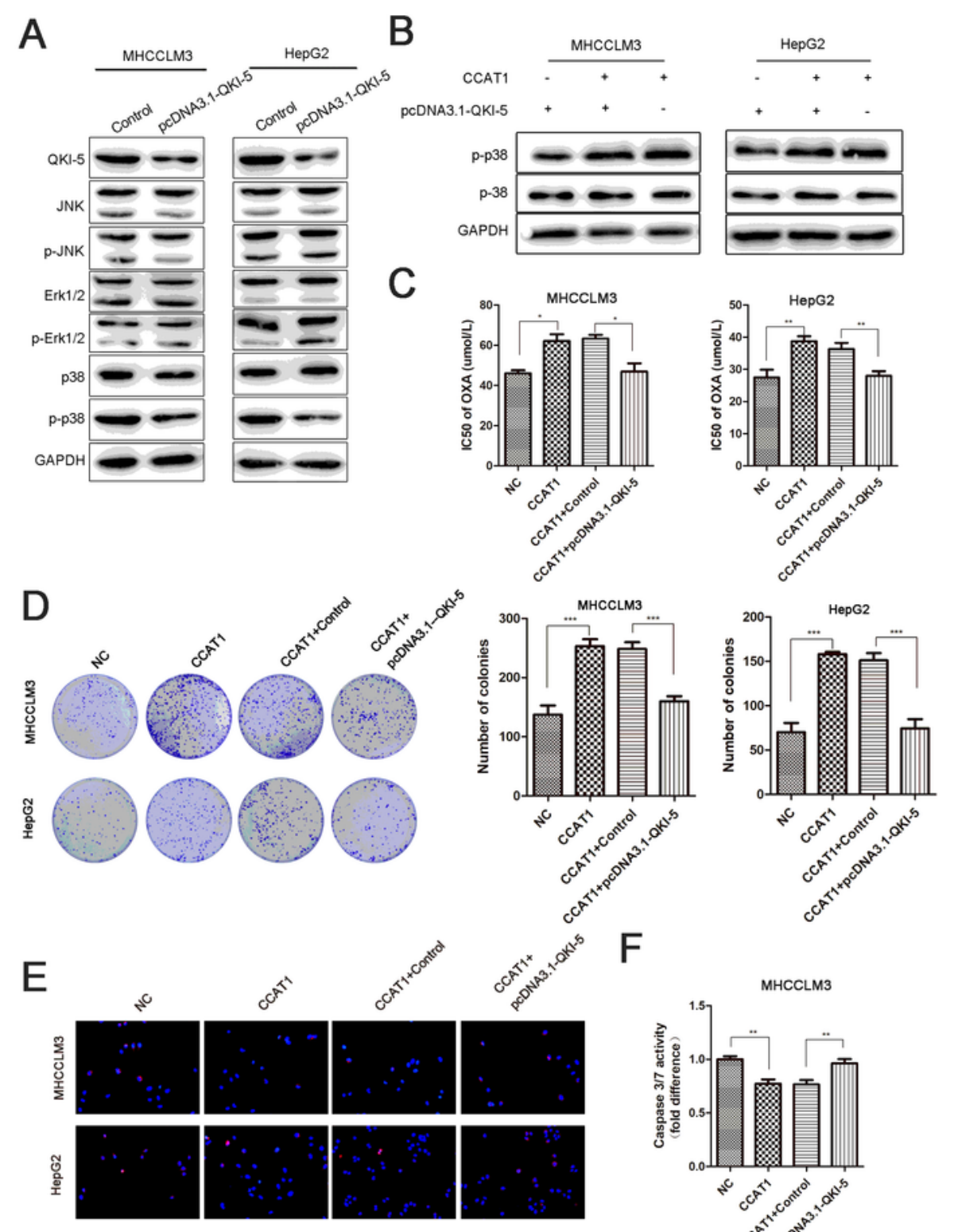

$\mathrm{F}$
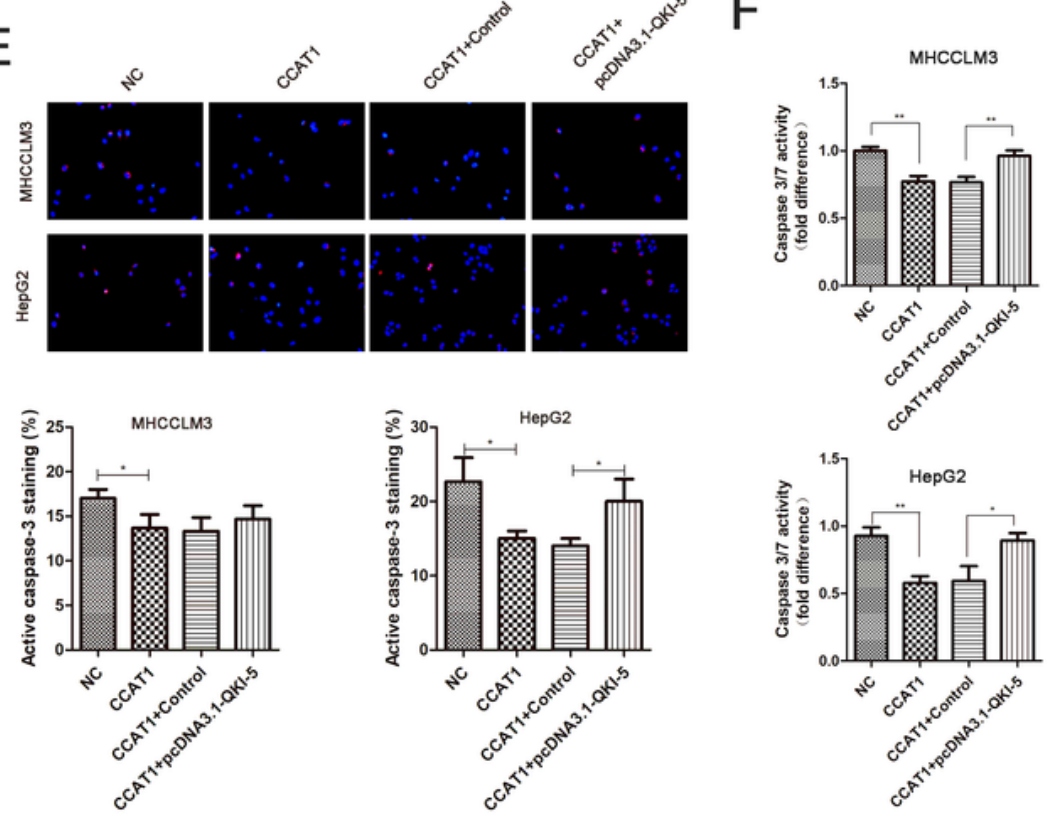

Figure 4 
Overexpression of QKI-5 reversed oxaliplatin resistance induced by CCAT1. (A) Western blotting analysis of expression levels of QKI-5, JNK, p-JNK, ERK1/2, p-ERK1/2, p38 and p-p38 MAPK in control and pcDNA3.1-QKI-5 cells. (B) The phosphorylation and total levels of p38 MAPK in the CCAT1 and QKI-5 overexpression HCC cells. (C) IC50 of oxaliplatin was determined when QKI-5 was upregulated. (D) The colony formation assay showed that overexpressed QKI-5 significantly attenuated cell proliferation induced by CCAT1 both in HCCLM3 and HepG2 cells. (E\&F) Fluorescence staining and Caspase-Glo® 3/7 Assay showed caspase-3 activity influenced by CCAT1 and QKI-5. Overexpressed QKI- 5 could increase caspase-3 activity reduced by CCAT1. *: $\mathrm{P}<0.05, * *: P<0.01, * \star *: P<0.001$.

\section{Supplementary Files}

This is a list of supplementary files associated with this preprint. Click to download.

- Tables1.doc

- Tables1.doc 